\title{
Assessment of chronic allograft injury in renal transplantation using diffusional kurtosis imaging
}

Xin Zheng ${ }^{1+}$, Min Li ${ }^{2+}$, Pan Wang ${ }^{2}$, Xiangnan $\mathrm{Li}^{2}$, Qiang Zhang ${ }^{3,4}$, Song Zeng ${ }^{3,4}$, Tao Jiang ${ }^{2 *}$ and Xiaopeng $\mathrm{Hu}^{3,4^{*}}$ (D)

\begin{abstract}
Background: Chronic allograft injury (CAl) is a significant reason for which many grafts were lost. The study was conducted to assess the usefulness of diffusional kurtosis imaging (DKI) technology in the non-invasive assessment of CAl.

Methods: Between February 2019 and October 2019, 110 renal allograft recipients were included to analyze relevant DKI parameters. According to estimated glomerular filtration rate (eGFR) $\left(\mathrm{mL} / \mathrm{min} / 1.73 \mathrm{~m}^{2}\right)$ level, they were divided to 3 groups: group 1, eGFR $\geq 60(n=10)$; group 2, eGFR 30-60 $(n=69)$; group 3, eGFR $<30(n=31)$. We performed DKI on a clinical 3T magnetic resonance imaging system. We measured the area of interest to determine the mean kurtosis (MK), mean diffusivity (MD), and apparent diffusion coefficient (ADC) of the renal cortex and medulla. We performed a Pearson correlation analysis to determine the relationship between eGFR and the DKI parameters. We used the receiver operating characteristic curve to estimate the predicted values of DKI parameters in the CAl evaluation. We randomly selected five patients from group 2 for biopsy to confirm CAl.

Results: With the increase of creatinine, ADC, and MD of the cortex and medulla decrease, MK of the cortex and medulla gradually increase. Among the three different eGFR groups, significant differences were found in cortical and medullary MK $(P=0.039, P<0.001, P<0.001$, respectively). Cortical and medullary ADC and MD are negatively correlated with eGFR $(r=-0.49,-0.44,-0.57,-0.57$, respectively; $P<0.001)$, while cortical and medullary MK are positively correlated with eGFR $(r=0.42,0.38 ; P<0.001)$. When 0.491 was set as the cutoff value, MK's CAl assessment showed $87 \%$ sensitivity and $100 \%$ specificity. All five patients randomly selected for biopsy from the second group confirmed glomerulosclerosis and tubular atrophy/interstitial fibrosis.
\end{abstract}

Conclusion: The DKI technique is related to eGFR as allograft injury progresses and is expected to become a potential non-invasive method for evaluating CAI.

Keywords: Diffusion-MRI, Non-Gaussian diffusion, DKI, Renal transplantation

*Correspondence: jiangt8166@hotmail.com; Xiaopeng_hu@sina.com ${ }^{\dagger}$ Xin Zheng and Min Li have contributed equally to this work. ${ }^{2}$ Department of Radiology, Beijing Chao-Yang Hospital, Capital Medical University, 8 Gongren Tiyuchang Nanlu, Chaoyang District, Beijing 200020, People's Republic of China

${ }^{3}$ Institute of Urology, Capital Medical University, 8 Gongren Tiyuchang Nanlu, Chaoyang District, Beijing 200020, People's Republic of China

Full list of author information is available at the end of the article

\section{Background}

For patients with end-stage renal disease, kidney transplantation may be the best treatment option. When the dialysis method is different, kidney transplantation can provide unparalleled results, such as survival rate, personal satisfaction, quality of life, and cost suitability [1]. 
Despite advances in surgical methods and immunosuppressants, the long-term effects of kidney allografts have not changed significantly in the last two decades. Chronic allograft injury (CAI) is the most common cause of kidney allograft failure, which can lead to a certain degree of delay.

The characteristics of CAI are glomerulosclerosis, tubular atrophy, vascular occlusive changes, and interstitial fibrosis. Early detection and exact CAI evaluation are critical to manage treatment and postpone or prevent irreversible damage to the transplanted renal [2,3].

Current methods for evaluating CAI have significant impediments. The most widely used techniques for testing allograft function are serum creatinine levels and estimated glomerular filtration rate (eGFR). However, they are believed to be affected by many factors, and their prognostic value for allograft injury is poor. When serum creatinine levels rise or eGFR falls, allograft damage may have progressed to the point that it is no longer reversible [4]. Accordingly, allograft biopsy was considered the golden choice to analyze allograft injury and differentiate among the different etiologies, despite its impediments, such as infection, bleeding, and even allograft loss [5]. However, the regular evaluation of CAI still can not be exactly. Like the eGFR might not be sensitive enough to evaluate CAI change, and the standard golden biopsy also seems to have significant risks. Hence, a critical need is needed to find non-invasive and precise techniques for diagnosing CAI to guide timely intervention.

The morpholoy, microstructue, and functional characteristics of renal allografts have been confirmed using magnetic resonance imaging (MRI). Several pieces of research have found a significant correlation between MRI and eGFR, like diffusion-weighted imaging (DWI), diffusion tensor imaging (DTI), blood-oxygen-leveldependent (BOLD) imaging, and arterial spin labeling (ASL), which indicated the potential of using MRI as a non-invasive biomarker [6-10]. MRI does not use ionizing radiation, allowing for repeated imaging after the patient has accepted a kidney transplant $[7,11]$.

Diffusional kurtosis imaging (DKI) is an extension of the traditional diffusional kurtosis imaging (DTI) model that takes into account non-microstructural complexity and diffusional heterogeneity. DKI needs at least three b-values, with the highest b-value exceeding DWI. Although standard DTI metrics like mean diffusivity (MD) and apparent diffusion coefficient (ADC) are available, DKI can provide parameters defined by mean kurtosis (MK). Among these parameters, MK is the significant one obtained from DKI, which renal allografts are believed to be a natural complexity. The degree of diffusion restriction on the non-Gaussian distribution of water molecules increases with the complexity of the structure and the MK value [12]. As a result, we hypothesized that the DKI technique could be associated with eGFR and could show microstructural changes in CAI more specifically than eGFR, and that the DKI technique could be a non-invasive tool for assessing CAI.

This study is planned to evaluate the correlation between eGFR and DKI parameters and the potential feasibility of the DKI technique in non-invasive assessment for CAI.

\section{Methods \\ Participants}

All the patients included in the analysis received kidney transplantation approved by the Institutional Review Board of Beijing Chao-Yang Hospital, Capital Medical University. They conformed to the tenets of the Declaration of Helsinki. Before allograft biopsy and functional MRI, written informed consent from all patients should be collected.

In this prospective single-center study conducted from February 2019 to October 2019, a total of 130 adult patients after kidney transplantation agreed to participate and had no MRI contraindications were inrolled. The existence of MRI-incompatible devices, claustrophobia, patient rejection, and a time span of less than three months between transplantation and MRI were all conditions for exclusion. When the MRI slot is available, select eligible patients who did not meet the exclusion criteria. The final analysis included only kidneys known to have been procured with the donors' consent or family members. The final report did not include data from patients who had kidney transplants from unknown sources.

Within one week of the biopsy, functional MRI examinations were performed. Blood samples were taken on the day of the MR test from all patients, and eGFR was determined by changing the renal disease equation [13]. Patients were divided into three groups based on allograft function: Group 1 had patients with appropriate allograft function $\left(e G F R>60\left(\mathrm{~mL} / \mathrm{min} / 1.73 \mathrm{~m}^{2}\right)\right.$, Group 2 had patients with mild allograft function $(30 \mathrm{~mL} /$ $\min / 1.73 \mathrm{~m}^{2}$ ), and Group 3 had patients with severely impaired renal function (eGFR30 $\left(\mathrm{mL} / \mathrm{min} / 1.73 \mathrm{~m}^{2}\right)$. Clinical status and renal function were monitored every 1-4 weeks in the first year after transplantation and every month after that. A minimum of 3 months of follow-up was available for all patients.

\section{MRI protocols}

All MRI examinations were performed on the 3T Prisma MRI scanner (Siemens Healthineers, Erlangen, Germany) using a 16-channel phased-array coil positioned over the pelvis. The anatomic images were obtained using transverse $\mathrm{T} 1$-weighted imaging $(\mathrm{TR} / \mathrm{TE}=700 / 12 \mathrm{~ms}$, 
slice thickness $=5.0 \mathrm{~mm}$, slice spacing $=0 \mathrm{~mm}$, FOV $=240 \mathrm{~mm}^{2}$, matrix size $\left.=256 \times 256\right)$ and transverse $\mathrm{T} 2$-weighted imaging $(\mathrm{TR} / \mathrm{TE}=3770 / 101 \mathrm{~ms}$, slice thickness $=5.0 \mathrm{~mm}$, slice spacing $=0 \mathrm{~mm}, \mathrm{FOV}=240 \mathrm{~mm}^{2}$, matrix size $=320 \times 320$ ) covering the transplant kidney. Following the anatomic scans, DWI was performed with single-shot echo-planar imaging (EPI) in the transverse plane, with locations identical to those prescribed for the transverse T2-weighted imaging, using six $b$-values $\left(0_{4}, 400_{6}, 800_{8}, 1200_{10}, 2000_{10}\right.$, and $3000_{12} \mathrm{~s} / \mathrm{mm}^{2}$, where the subscript denotes the number of averages). The other key acquisition parameter for DWI were: TR/ $\mathrm{TE}=3100 / 68 \mathrm{~ms}$, slice thickness $=3.5 \mathrm{~mm}$, slice spacing $=0 \mathrm{~mm}$, matrix size $=120 \times 120$, FOV $=350 \mathrm{~mm}$, and the scan time $\sim 9 \mathrm{~min}$.

\section{Functional MRI image analyses}

All MR images were analyzed by two radiologists (with more than ten years of experience in abdominal imaging) who did not know the diagnosis or pathophysiologic grade. Regions of interest (ROI) were manually drawn on DWI with $b$ value $=0 \mathrm{~s} / \mathrm{mm}^{2}$ and then copied onto the corresponding position on all parameter maps. 18 ROIs were positioned on three parts (upper pole, central area, and lower pole) of the transplanted kidney: for each segment, three ROIs (anterior labrum, posterior labrum and, intermediate site) were drawn on the cortex and another three on the medulla. Finally, the values of ADC and DKI parameters ( $\mathrm{MK}$ and $\mathrm{MD}$ ) were calculated as an average of all voxels contributing to the ROI, and then did further quantitative analysis detailed below. Artifacts were avoided when placing ROIs. All measurements were repeated twice, and the values of $K, D$, and ADC were recorded.

\section{Histopathological analysis}

We performed biopsies randomly in patients of Group 2 to confirm the histopathological changes. The biopsy indicated eGFP $<60\left(\mathrm{~mL} / \mathrm{min} / 1.73 \mathrm{~m}^{2}\right)$ with or without gradual deterioration during the follow-up and patient consent. Kidney tissue was obtained by needle biopsy and fixed in $4 \%$ buffered formaldehyde. The tissue was then embedded in paraffin and serially sliced into $2-\mathrm{m}$ thick portions. To evaluate glomerulosclerosis and tubular atrophy, the parts were stained with hematoxylin and eosin (H\&E) and periodic acid silver methenamine (PASM), respectively, and Masson-trichrome Goldner's to measure interstitial fibrosis. The diagnosis procedures were performed according to the renal pathology laboratory's standard protocols, as reported previously [14]. Two qualified neuropathologists with 15 years and 18 years of experience, respectively, conductedd pathologic diagnoses according to the Banff 2015 scheme [15] without referring to functional MRI results.

\section{The inter-reader agreement assessment}

We evaluated the repeatability of MRI parameters using interclass correlation coefficients(ICCs). The ICC was classified as good consistency when it was larger than 0.80 .

\section{Statistical analysis}

SPSS 24.0 for Windows (SPSS, Chicago, IL, USA) was used for statistical data analysis. We used the Kolmogorov-Smirnov test to testify the normality of the data. Variables that are normally distributed are represented as mean values with standard deviations. Categorical variables are described as a percentage. We used Two-tailed paired Student $t$-tests to compare cortical and medullary DKI parameters. A one-way analysis of variance (ANOVA) was performed to test the difference in ADC, Mean K, and Mean D values between the three groups. A Pearson correlation analysis was performed to determine the relationship between the MRI parameters and eGFR. The receiver operating characteristic (ROC) curve analysis of diffusional kurtosis parameters was performed to predict normal and mildly impaired renal function in the recipients to evaluate the diagnostic performances of diffusional kurtosis parameters in predicting impaired renal function without the server impaired renal function group. $P<0.05$ indicated that the difference was statistically significant.

\section{Results \\ Patient demographics, clinical characteristics, and laboratory characteristics}

A total of 130 patients were included in this cohort, and if there were MRI-incompatible devices, claustrophobia, and patient refusal $(n=20)$, they were excluded. Finally, the functional MRI data of 110 patients (mean age, $44.32 \pm 9.562$ years) were analyzed (Fig. 1), containing 82 males (mean age, $43.50 \pm 9.535$ years) and 28 females (mean age, $46.71 \pm 9.40$ years). The time interval between renal transplantation and MR examination was three months to 300 months, with a median of 42.29 months. No statistically significant difference was observed between these three groups about donor demographics, recipient age, sex, kidney transplant type, and immunosuppressive regimens used. (Table 1).

Unsurprisingly, the mean hemoglobin level of Group 3 was much lower than that of both Gourp1 and Group2.

\section{Diffusional Kurtosis imaging}

The corticomedullary difference is displayed by diffusional kurtosis imaging. (Fig. 2). 


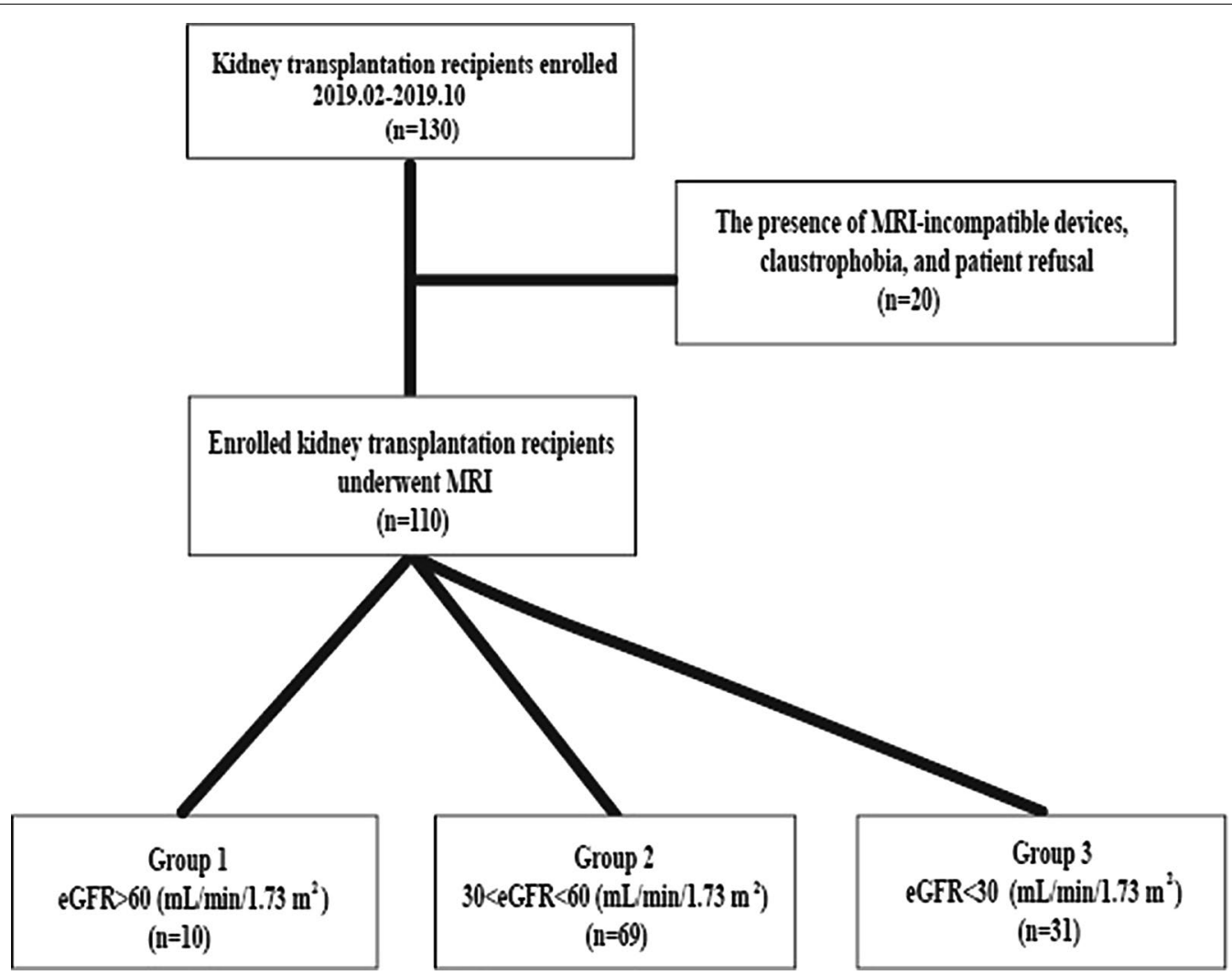

Fig. 1 Flowchart of patient enrollment, exclusion criteria, final study sample, and categorization of patients to different groups

Table 1 Patient demographics, clinical features, and laboratory characteristics in 110 patients who underwent functional magnetic resonance imaging

\begin{tabular}{|c|c|c|c|}
\hline Characteristics/findings & Group 1 & Group 2 & Group 3 \\
\hline No. of patients & 10 & 69 & 31 \\
\hline Sex (men: women) & $5: 5$ & $11: 58$ & $12: 19$ \\
\hline Age(months) & $50.4 \pm 5.4$ & $43.1 \pm 9.5$ & $44.9 \pm 10.3$ \\
\hline Time post transplantation(months) & $35 \pm 25.8$ & $31.96 \pm 26.1$ & $67.32 \pm 47.2$ \\
\hline \multicolumn{4}{|l|}{ Immunosuppressive regimens } \\
\hline Pre + MMF + FK506, n (\%) & $8(80)$ & $60(87)$ & $27(87.1)$ \\
\hline Pre + MMF + CsA, n (\%) & 0 & $6(8.7)$ & $2(6.5)$ \\
\hline Other, n (\%) & $2(20)$ & $3(4.3)$ & $2(6.5)$ \\
\hline Serum creatinine, $\mathrm{mg} / \mathrm{dl}$, mean $\pm \mathrm{SD}$ & $65.3 \pm 4.6$ & $108.8 \pm 33.6$ & $168.4 \pm 104.6$ \\
\hline $\mathrm{eGFR}, \mathrm{ml} / \mathrm{min} / 1.73 \mathrm{~m}^{2}$, mean $\pm \mathrm{SD}$ & $65.6 \pm 5.1$ & $41.7 \pm 8.8$ & $24.3 \pm 8.1$ \\
\hline Hemoglobin level, g/dl, mean \pm SD & $143.6 \pm 12.7$ & $145.26 \pm 20.5$ & $117 \pm 22.8$ \\
\hline
\end{tabular}

Pre Prednisone, MMF Mycophenolate mofetil

Table 2 demonstrated the value of different cortical and medullary DKI parameters in three groups. Except for the mean $\mathrm{D}$ of the medulla of group $3(P=0.277)$, the renal cortex parameters (including $\mathrm{MK}$ and $\mathrm{MD}$ values) of the subjects in all three groups were significantly higher than the medulla of the subjects $(P<0.05)$.
Nevertheless, the cortical ADC was lower than medullary ADC $(P<0.05)$. ADC and Mean D values of the renal cortex and medulla decreased gradually with the increase of creatinine. In contrast, the Mean $\mathrm{K}$ values of the renal cortex and medulla increased gradually with creatinine. 

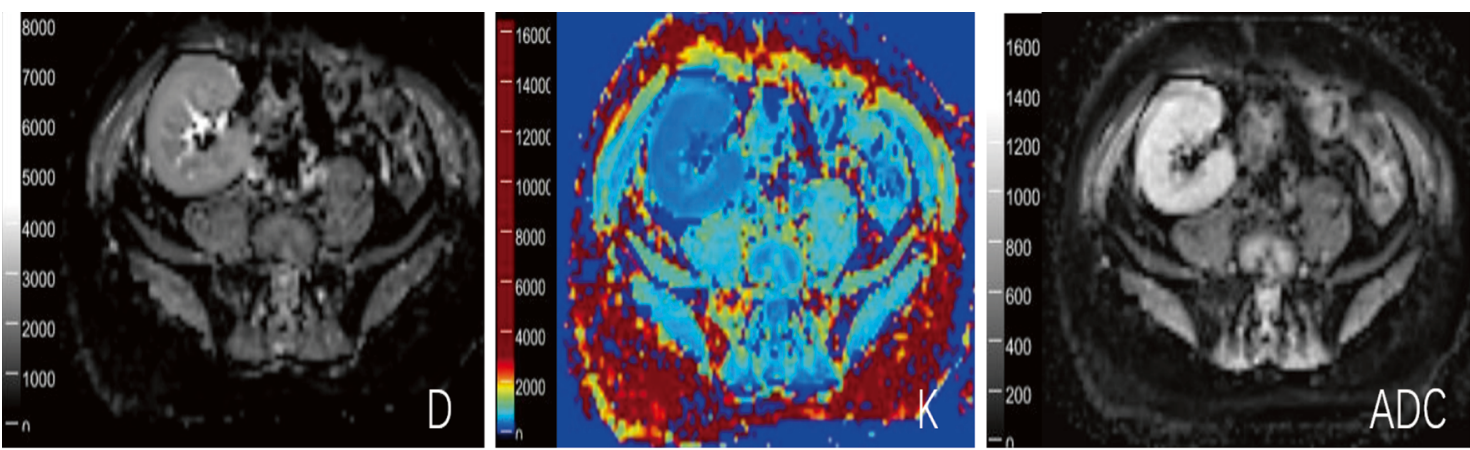

\section{Patient 1}
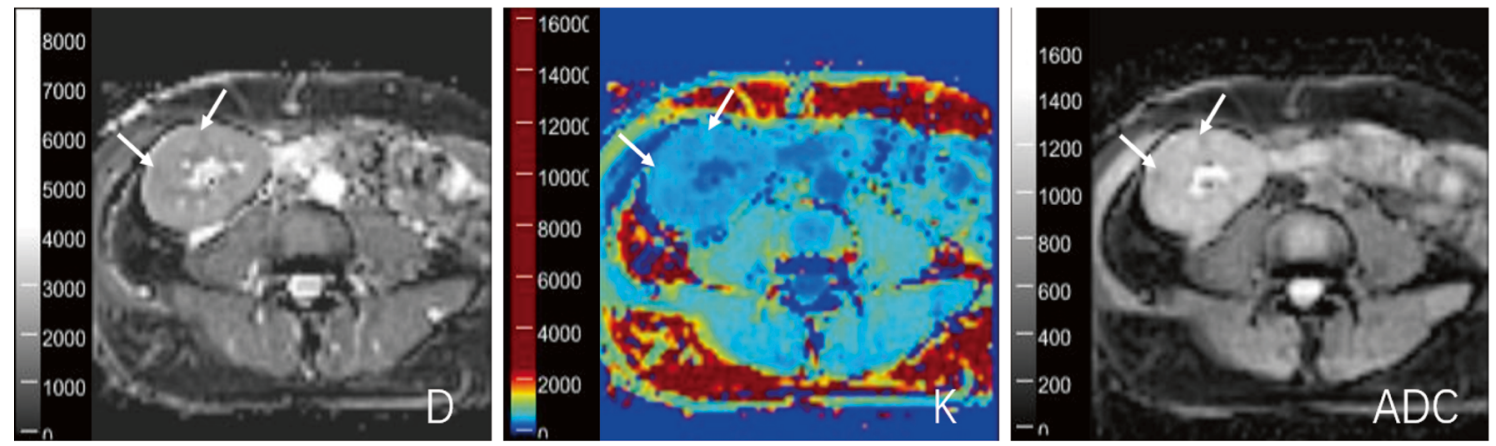

Patient 2
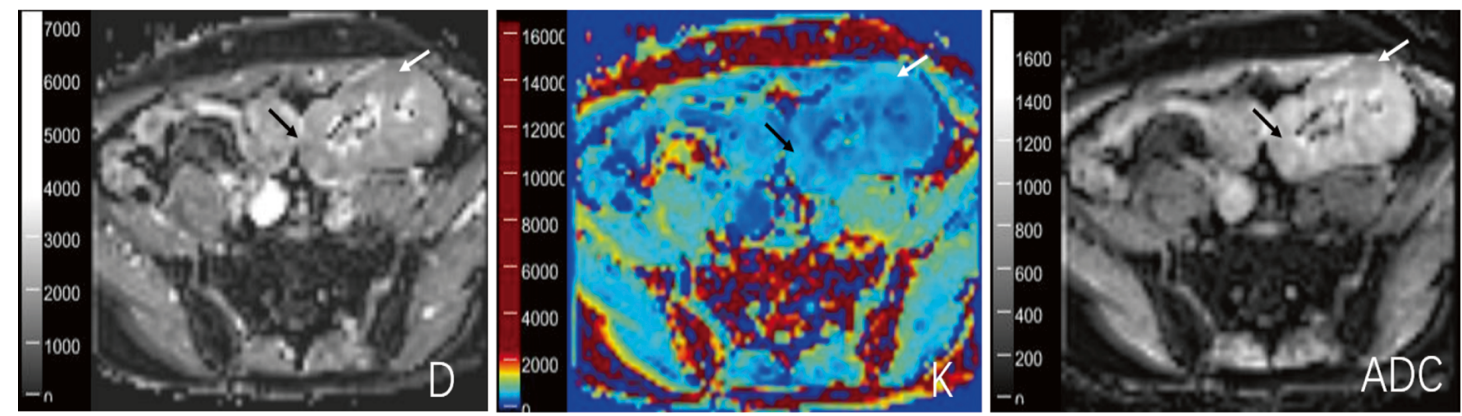

\section{Patient 3}

Fig. 2 The Diffusion kurtosis Imaging parameter maps (ADC map, K map, and D map) of three patients with different eGFR levels. Patient 1 , $\mathrm{eGFR}=71.57$. The $\mathrm{D}, \mathrm{K}$, and ADC map showed that the renal allograft parenchyma had evident signal. Patient 2, eGFR $=47.48$. The D and ADC map showed multiple little patchy hypointensive signals in the renal allograft parenchuma, while the K map showed multiple little patchy hyperintensive signals. (as depicted by the arrows). Patient 3, eGFR $=21.49$. The D and ADC map showed diffuse low signal intensity in the renal allograft parenchuma, while the K map showed diffuse high signal intensity. (as depicted by the arrows)

As showed in Fig. 3, all parameters showed significant differences $(P<0.05)$ except Cortical ADC and Medullary Mean D between Group 1 and Group $2(P=0.117$, $P=0.257$, respectively).

\section{Correlations of DKI parameters with eGFR}

The Mean $\mathrm{K}$ of renal cortex and medulla of all the patients showed positive correlations with eGFR $(r=0.42,0.38$; $P<0.001)$, while the ADC and MD of renal cortex and medulla all correlated negatively with eGFR $(\mathrm{r}=-0.49$, $-0.44,-0.57,-0.57$, respectively; $P<0.001$ ). (Fig. 4 ).

\section{ROC analysis results}

In each of these six parameters, the Mean $\mathrm{K}$ of the renal cortex showed the most prominent area under the stove of 0.967 . In contrast, the medullary Mean $\mathrm{K}$ showed a comparable area under the curve of 0.960 . When 0.491 was set as the cutoff value, cortical mean K's sensitivity 
Table 2 Comparison of diffusional kurtosis parameters of renal cortex and medulla in each group

\begin{tabular}{|c|c|c|c|c|c|c|}
\hline & \multicolumn{2}{|c|}{$\operatorname{ADC}\left(10^{-3} \mathrm{~mm}^{2} / \mathrm{s}\right)$} & \multicolumn{2}{|c|}{ Kurtosis $\left(10^{-3} \mathrm{~mm}^{2} / \mathrm{s}\right)$} & \multicolumn{2}{|l|}{$\mathrm{D}\left(10^{-3} \mathrm{~mm}^{2} / \mathrm{s}\right)$} \\
\hline & Cortex & Medulla & Cortex & Medulla & Cortex & Medulla \\
\hline Group 1 & $\begin{array}{l}1.192 \pm 0.049 \\
(P=0.000)\end{array}$ & $1.236 \pm 0.063$ & $\begin{array}{l}0.470 \pm 0.013 \\
(P=0.039)\end{array}$ & $0.458 \pm 0.017$ & $\begin{array}{l}2.778 \pm 0.409 \\
(P=0.000)\end{array}$ & $2.625 \pm 0.377$ \\
\hline Group 2 & $\begin{array}{l}1.141 \pm 0.097 \\
(P=0.25)\end{array}$ & $1.155 \pm 0.116$ & $\begin{array}{l}0.521 \pm 0.044 \\
(P=0.000)\end{array}$ & $0.511 \pm 0.041$ & $\begin{array}{l}2.585 \pm 0.237 \\
(P=0.001)\end{array}$ & $2.533 \pm 0.230$ \\
\hline Group 3 & $\begin{array}{l}1.016 \pm 0.116 \\
(P=0.002)\end{array}$ & $1.049 \pm 0.105$ & $\begin{array}{l}0.554 \pm 0.042 \\
(P=0.000)\end{array}$ & $0.535 \pm 0.039$ & $\begin{array}{l}2.183 \pm 0.323 \\
(P=0.27)\end{array}$ & $2.178 \pm 0.197$ \\
\hline
\end{tabular}

Statistical analysis was employed using the t-test. Data presented as mean \pm SD. A $P<0.05$ was considered statistically significant

to predicting normal and mild impaired renal function is $87 \%$, and the specificity is $100 \%$. Meanwhile, When 0.499 was set as the cutoff value, the medullary Mean D demonstrated a sensitivity of $61 \%$ and specificity of $100 \%$ for predicting normal and mildly impaired renal function. (Fig. 5 and Table 3).

\section{The results of inter-reader agreement assessment}

The results of the ICCs were displayed in the Additional file 1: Table 1 . The results of ICCs ranged from 0.83 to 0.99 , representing fair agreements for the measurement data.

\section{Pathology findings}

We randomly selected five patients from group 2 to confirm the diagnose of CAI. The five patients' primary pathologic findings were glomerulosclerosis and tubular atrophy/interstitial fibrosis, which conform to CAI diagnosis (Fig. 6).

\section{Discussion}

The present study first proposed the non-Gaussian DKI model as a potential non-invasive method for evaluating CAI.

Several new advances in MRI have enabled the noninvasive assessment of allograft. As reviewed by Ljimani et al. [7], with the development of MRI, multiple of MRI parameters showed the potential of assessment of allograft function. As first investigated in dynamic contrastenhanced (DCE) [16],parameters likeDWI [17, 18], DTI $[6,19,20]$, ASL $[21,22]$, BOLD [23, 24] then were also used for allograft renal imaging to assess the allograft function. Jensen et al. first proposed DKI in 2005 [25], as an extension of conventional DWI, which requires ultrahigh b-values $\left(>1000 \mathrm{~s} / \mathrm{mm}^{2}\right)$ and a modified image post-processing procedure. The traditional model of DWI was established based on the assumption that water diffusion exhibits Gaussian behavior without any restriction and that the diffusion-weighted MRI signal mono-exponentially decreases with increasing b-values; however, a deviation from simple mono-exponential decay is readily identified in the kidney, under either healthy or pathological conditions [26]. DKI could be used to investugate non-Gaussian diffusion of water with a polynomial model and has been used to identify the heterogeneity of cellularity and microstructural complexity $[27,28]$.

DKI can yield two characteristic variables: D and K. D is the diffusion coefficient corrected by a non-Gaussian bias, and $\mathrm{K}$ quantifies the deviation of tissue diffusion from a Gaussian pattern [25, 28]. Recently, in animal models, DKI has been used to assess liver fibrosis [29, 30], which demonstrated additional meaningful information different from that of conventional DWI. Only two studies focused on DKI in healthy kidneys, which showed conflicting results [31, 32]. Furthermore, the findings revealed that the question of whether the maximal b-values $(600$ and $1000 \mathrm{~s} / \mathrm{mm})$ are sufficiently high remains debatable. Huang et al. [31] showed that in a normally functioning kidney, the cortex's MK value is lower than that of the medulla. Among these diffusion kurtosis indicators, the difference between cortex and medulla is reliable with the presence of radially-oriented vessels, tubules, and collecting ducts in the medulla [31]. Interestingly, Pentang et al. [32] showed that the cortical MK is larger than the medullary MK.

As a particular metric of the DKI model, $\mathrm{K}$ has been hypothesized to represent the direct interaction of water molecules with the cell membrane intracellular compounds, and expanded $\mathrm{K}$ recommends that it has increasingly irregular and heterogeneous environments with numerous great interfaces. In tumor cells, an increased nuclear-cytoplasmic ratio and microstructural were revealed by $\mathrm{K}$ value $[27,33]$. The precise basic meaning of diffusional kurtosis metrics has yet been grasped, and DKI acquisition has not yet been perfected.

Liu et al. [34] found that in the pathogenesis of IgAN, the progressive loss of glomerular capillary structures 


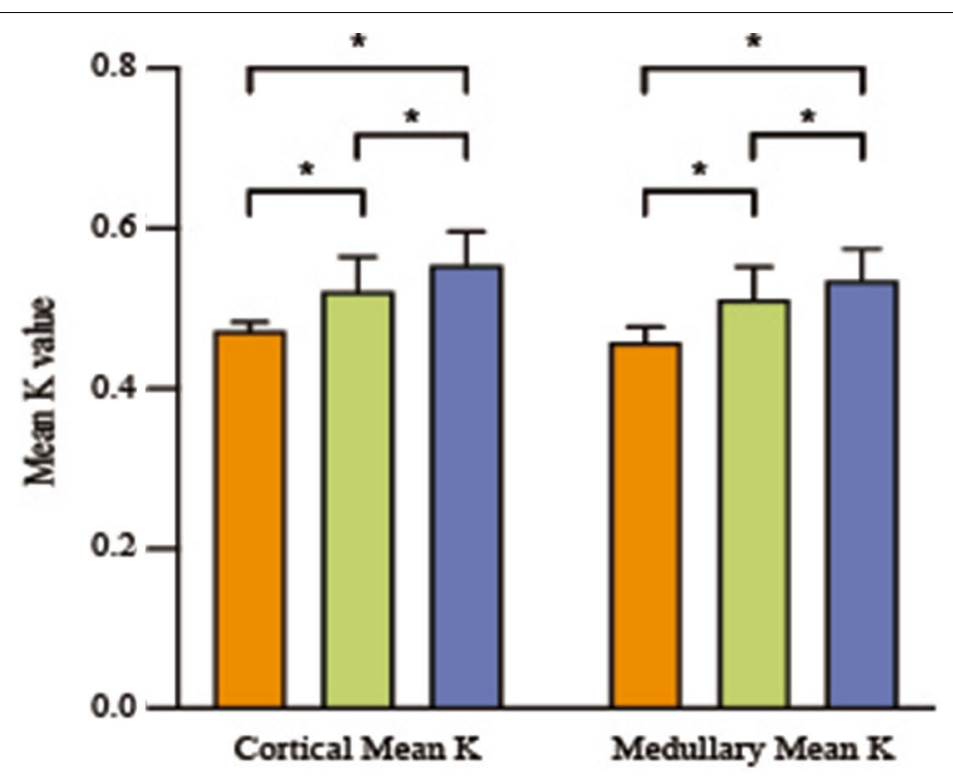

a

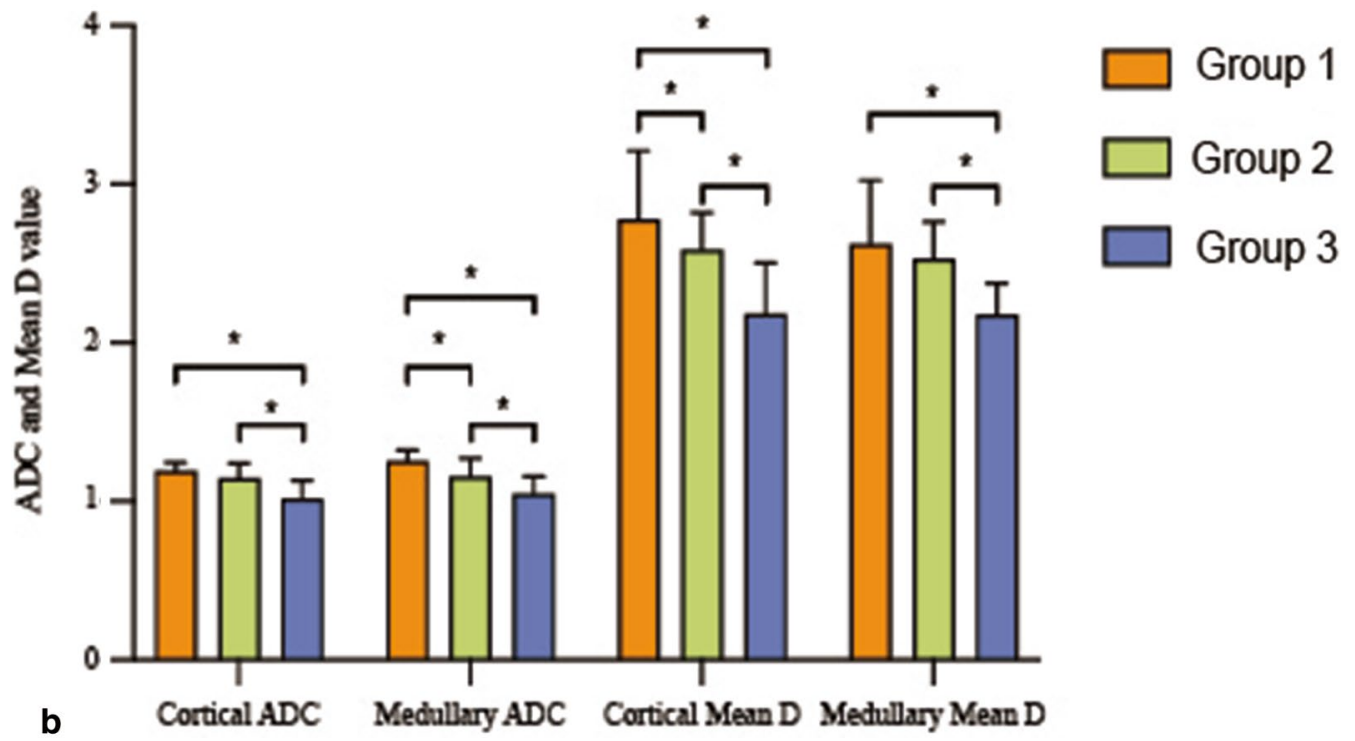

Fig. 3 Comparisons of cortical and medullary diffusion kurtosis parameters between different groups. a Cortical and Medullary Mean $\mathrm{K}$ differ significantly among eGFR levels $(P<0.001)$. b ADC and Mean D of both cortex and Medulla differ significantly among eGFR levels $(P<0.001)$, except Cortical ADC and Medullary Mean D between Group 1 and Group 2

and the disappearance of glomerular cellular elements with replacement by an expanding extracellular matrix and fibrous tissue could result in more complex microstructure and marked variation in cell size and shape than in healthy kidneys, leading to increased $\mathrm{K}$.

Our study found that the MK increased gradually with the deterioration of kidney function, which indicates the increase of a much more irregular and heterogeneous environment in renal allograft with the worsening of renal function. As we know, the primary pathology change of CAI is glomerulosclerosis and tubular atrophy/ interstitial fibrosis, which means a tendency of the more irregular and heterogeneous environment in the renal allograft.

Liu et al. also demonstrated that $\mathrm{K}$ showed better performance than ADC in glomerulosclerosis in terms of diagnostic efficacy, with a relatively larger AUC and stronger correlation. However, the level of statistical significance was not achieved [34]. These results indicate that the K in the DKI model showed clinical potential for 


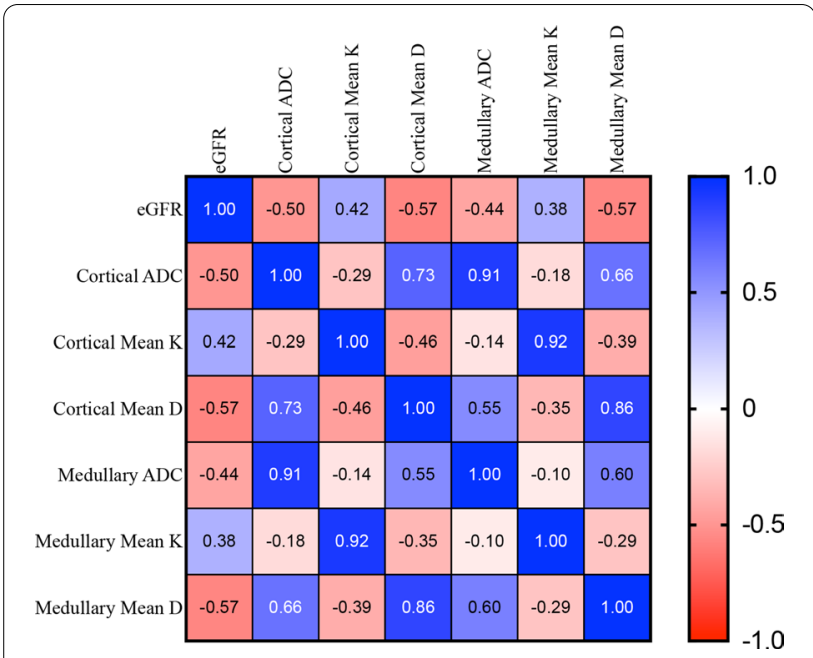

Fig. 4 Correlation analysis results between eGFR and diffusion kurtosis parameters $(P<0.05)$

assessing the severity of renal sclerosis in the glomeruli and providing more information than ADC.

Our study found that ADC and the Mean D value of cortex in patients with severely decreased eGFR were significantly lower than those in higher eGFR. In comparison, the Mean $\mathrm{K}$ value in patients with higher eGFR was lower than in patients with severely reduced eGFR.

According to our research, although all the six parameters showed significant differences except cortical ADC and medullary Mean D between Group 1 and Group 2, the ROC's considerable differences were only found in ADC of Medulla and MK of cortex and medulla. However, the ADC demonstrated an extremely low specificity, and the MK of the cortex showed the largest AUC. Meanwhile, we performed a random autopsy to confirm the histography change of renal allograft. We found glomerulosclerosis and tubular atrophy/interstitial fibrosis of the randomly selected patients, which demonstrated that $\mathrm{K}$ increased with the deterioration of renal function and renal fibrosis progression. We suggested that Mean $\mathrm{K}$ showed excellent CAI prediction for identifying both glomerulosclerosis and tubular atrophy/interstitial fibrosis.

Simultaneously, the higher Mean $\mathrm{K}$ value of patients with decreased renal function may be partially due to interstitial fibrosis. The higher cell density and collagen deposition may result in lower ADC values in renal allografts [35]. This characteristic showed that DKI parameters have broad clinical application prospects in the non-invasive screening of renal allografts' function at

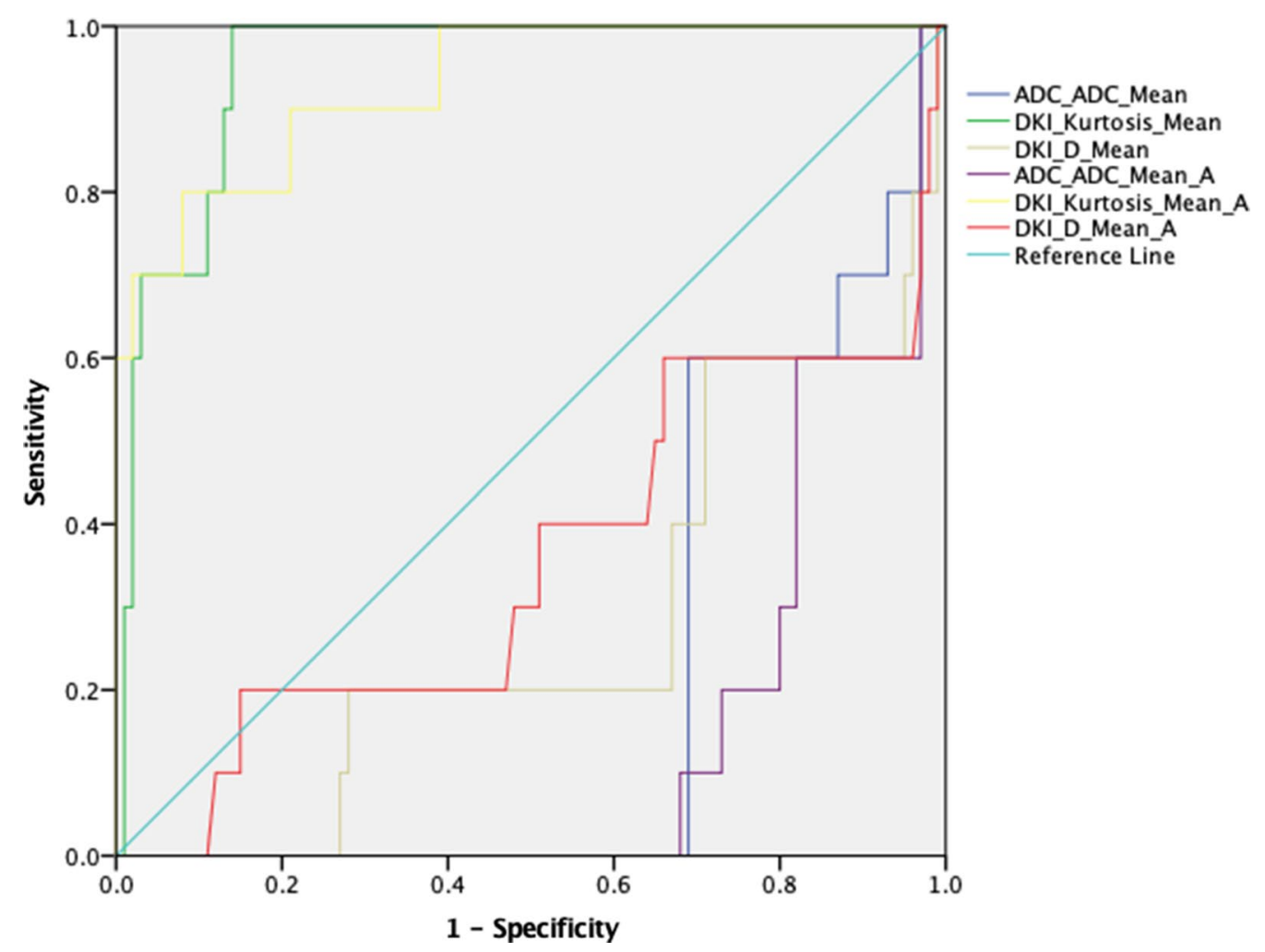

Fig. 5 Receiver operating characteristic curves of diffusion kurtosis parameters 
Table 3 Performances of diffusion kurtosis imaging parameters in predicting decreased transplanted kidney function

\begin{tabular}{|c|c|c|c|c|c|c|}
\hline \multirow[t]{2}{*}{ Index } & \multicolumn{2}{|c|}{$\operatorname{ADC}\left(10^{-3} \mathrm{~mm}^{2} / \mathrm{s}\right)$} & \multicolumn{2}{|c|}{ Kurtosis $\left(10^{-3} \mathrm{~mm}^{2} / \mathrm{s}\right)$} & \multicolumn{2}{|c|}{$\mathrm{D}\left(10^{-3} \mathrm{~mm}^{2} / \mathrm{s}\right)$} \\
\hline & Cortex & Medulla & Cortex & Medulla & Cortex & Medulla \\
\hline AUC & 0.218 & 0.142 & 0.967 & 0.960 & 0.282 & 0.344 \\
\hline Cut off value & & 1.429 & 0.491 & 0.499 & & \\
\hline Sensitivity\% & & $100 \%$ & $87 \%$ & $61 \%$ & & \\
\hline Specificity\% & & $3 \%$ & $100 \%$ & $100 \%$ & & \\
\hline$P$ value & $(P=0.051)$ & $(P=0.042)$ & $(P=<0.022)$ & $(P=<0.041)$ & $(P=0.085)$ & $(P=0.104)$ \\
\hline
\end{tabular}

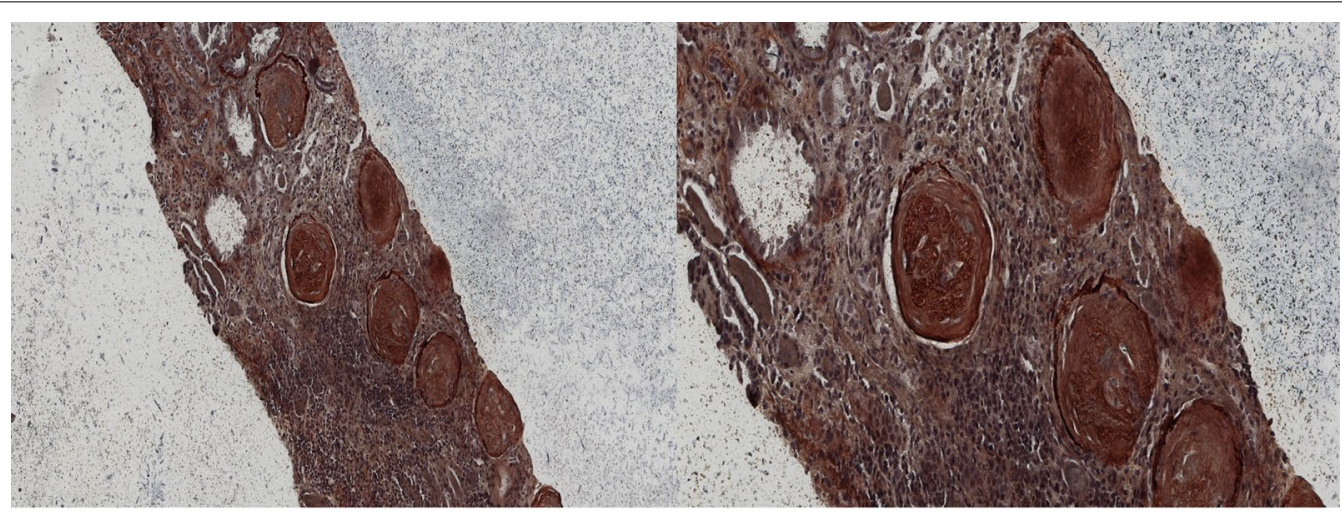

\section{Patient 1}
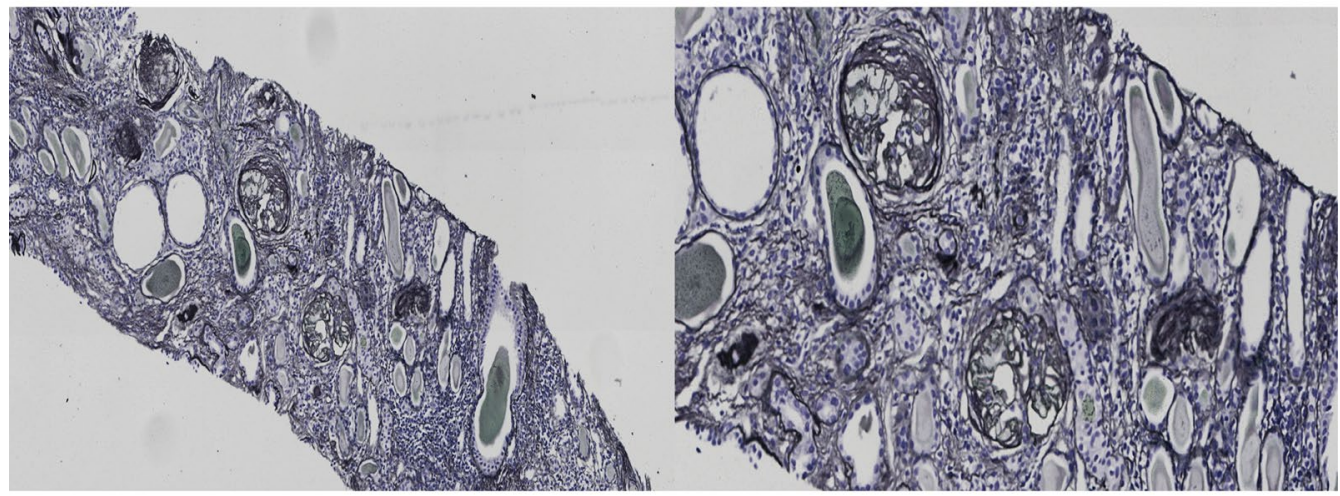

Patient 2

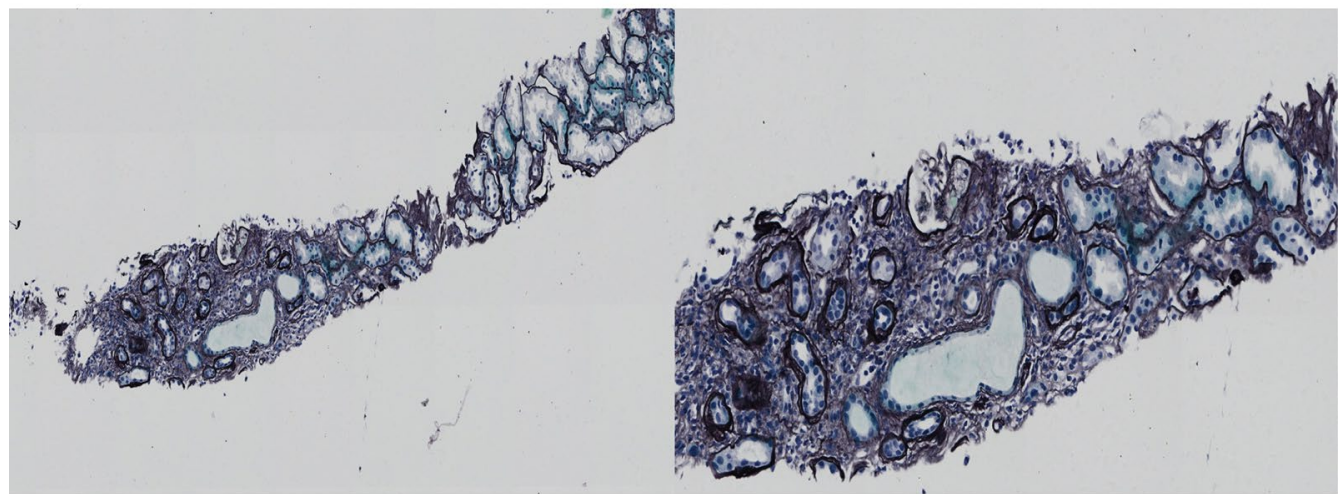

Patient 3

Fig. 6 Pathological images of three patients with different eGFR levels renal pathology (PASM *20and *40) 
various stages. The Mean $\mathrm{K}$ in the cortex had a sensitivity of $87 \%$ and a specificity of $100 \%$ when 0.491 was used as the cutoff value for predicting impaired allograft activity..

There are limitations to this study. First, the number of patients with normal eGFR was small. Moreover, it limits the accuracy of ROC curves. Second, not all the patients were performed biopsy in this study to analyze the quantitative correlation between histopathologic results and DKI parameters.

So, although we confirmed that the DKI model was associated with the changes of eGFR and can assess CAI to some extent, if the DKI model can evaluate CAI evolution more accurately before the change of eGFR, it still needs to be studied. In the future, we aim to perform a more extensive sample size research to explore if the micro-changes detected by the DKI model can stand for the change of CAI more accurately, even earlier than eGFR.

\section{Conclusions}

In conclusion, the non-invasive KI model was closely associated with the eGFR as allograft injury progresses, as renal perfusion might be reduced. The parameter MK of the renal cortex can non-invasively assess CAI to some extent. The DKI technique is correlated with eGFR and can be expected to be a non-invasive method to evaluate CAI potentially.

\section{Abbreviations}

ADC: Apparent diffusion coefficient; MK: Mean kurtosis; MD: Mean diffusivity; DTI: Diffusion tensor imaging; eGFR: Estimated glomerular filtration rate; CAl: Chronic allograft injury; MRI: Magnetic resonance imaging; ROC: Receiver operating characteristic.

\section{Supplementary Information}

The online version contains supplementary material available at https://doi. org/10.1186/s12880-021-00595-3.

Additional file 1: Table 1. The ICCs of two observers on multiple parameters.

\section{Acknowledgements}

The original version of the abstract was published in the poster of abstracts of American Transplant Conggress 2020 [36].

\section{Authors' contributions}

Guarantors of the integrity of the entire study, TJ, XPH; study concepts/study design or data acquisition or data analysis/interpretation, all authors; manuscript drafting or manuscript revision for important intellectual content, all authors; approval of the final version of the submitted manuscript, all authors; agrees to ensure any questions related to the work are appropriately resolved, all authors; literature research, XZ, ML, TJ, XPH; clinical studies, XZ, ML, TJ, $\mathrm{XPH}$; MRI studies, XZ, ML, PW, XNL; statistical analysis, XZ. ML; and manuscript editing, XZ, ML, QZ, SZ, TJ, XPH. As the article is a comprehensive work of both Urology and Radiology, it needs the cooperation of both departments. Dr. XZ and Dr. ML from Urology and Radiology, separately, cooperated very well in all the processes of this research. Dr. XZ attributed more work regarding collecting clinical data, while Dr. ML attributed more work regarding the collection of
MRI data. Furthermore, Dr. XZ wrote the clinical part of the article, and Dr. ML wrote the piece on MRI protocols. Then they incorporated it in the discussion parts of the article. So, we think they attributed equal to the article and should be the co-first author. All authors read and approved the final manuscript.

\section{Funding}

This study has received funding from the National Natural Scientific Foundation, China, to Xiaopeng Hu (Grant No.: 81970645, 81670679).

\section{Availability of data and material}

All the data and materials can be offered if required. Xin Zheng (mike. zheng@163.com) can be contacted for the requesting of data from this study.

\section{Declarations}

\section{Ethics approval and consent to participate}

This study was approved by the Institutional Review Board of Beijing ChaoYang Hospital, Capital Medical University. All procedures performed in this study were in accordance with the ethical standards of the institutional and/ or national research committee and with the 1964 Helsinki declaration and its later amendments or comparable ethical standards. Written informed consent was gathered from all patients before the functional MRI and allograft biopsies.

\section{Consent for publication}

Not applicable.

\section{Competing interests}

The authors have no competing interests.

\section{Author details}

1 Department of Urology, Beijing Youan Hospital, Capital Medical University, No. 8, Xi Tou Tiao, Youanmen Wai, Fengtai District, Beijing 100069, People's Republic of China. ${ }^{2}$ Department of Radiology, Beijing Chao-Yang Hospital, Capital Medical University, 8 Gongren Tiyuchang Nanlu, Chaoyang District, Beijing 200020, People's Republic of China. ${ }^{3}$ Institute of Urology, Capital Medical University, 8 Gongren Tiyuchang Nanlu, Chaoyang District, Beijing 200020, People's Republic of China. ${ }^{4}$ Department of Urology, Beijing Chao-Yang Hospital, Capital Medical University, 8 Gongren Tiyuchang Nanlu, Chaoyang District, Beijing 200020, People's Republic of China.

Received: 27 September 2020 Accepted: 28 March 2021

Published online: 07 April 2021

\section{References}

1. Cavallo MC, Sepe V, Conte F, et al. Cost-effectiveness of kidney transplantation from DCD in Italy. Transpl Proc. 2014;46:3289-96. https://doi.org/ 10.1016/j.transproceed.2014.09.146.

2. Aubert O, Kamar N, Vernerey D, et al. Long term outcomes of transplantation using kidneys from expanded criteria donors: prospective, population based cohort study. BMJ. 2015;351:h3557. https://doi.org/10.1136/ bmj.h3557.

3. Goldberg RJ, Weng FL, Kandula P. Acute and chronic allograft dysfunction in kidney transplant recipients. Med Clin N Am. 2016;100:487-503. https://doi.org/10.1016/.mcna.2016.01.002.

4. Earley A, Miskulin D, Lamb EJ, et al (2012) Estimating equations for glomerular filtration rate in the era of creatinine standardization: a systematic review. Ann Intern Med 156:785-795, W-270, W-271, W-272, W-273, W-274, W-275, W-276, W-277, W-278. https://doi.org/https://doi. org/10.7326/0003-4819-156-6-201203200-00391

5. Giral M, Renaudin K, Naesens M, et al. The 1-year Renal Biopsy Index: a scoring system to drive biopsy indication at 1-year post-kidney transplantation. Transpl Int Off J Eur Soc Organ Transpl. 2018. https://doi.org/10. 1111/tri.13290.

6. Lanzman RS, Ljimani A, Pentang G, et al. Kidney transplant: functional assessment with diffusion-tensor MR imaging at 3T. Radiology. 2013;266:218-25. https://doi.org/10.1148/radiol.12112522. 
7. Ljimani A, Wittsack H-J, Lanzman RS. Functional MRI in transplanted kidneys. Abdom Radiol N Y. 2018;43:2615-24. https://doi.org/10.1007/ s00261-018-1563-7.

8. Mathys C, Blondin D, Wittsack H-J, et al. T2' imaging of native kidneys and renal allografts-a feasibility study. ROFO Fortschr Geb Rontgenstr Nuklearmed. 2011;183:112-9. https://doi.org/10.1055/s-0029-1245597.

9. Heusch P, Wittsack H-J, Blondin D, et al. Functional evaluation of transplanted kidneys using arterial spin labeling MRI. J Magn Reson Imaging JMRI. 2014:40:84-9. https://doi.org/10.1002/jmri.24336.

10. Hueper K, Gueler F, Brasen JH, et al. Functional MRI detects perfusion impairment in renal allografts with delayed graft function. Am J Physiol Ren Physiol. 2015;308:F1444-51. https://doi.org/10.1152/ajprenal.00064. 2015.

11. Wang Y-T, Li Y-C, Yin L-L, et al. Functional assessment of transplanted kidneys with magnetic resonance imaging. World J Radiol. 2015;7:343-9. https://doi.org/10.4329/wjr.v7.i10.343.

12. Yu YM, Ni QQ, Wang ZJ, et al. Multiparametric functional magnetic resonance imaging for evaluating renal allograft injury. Korean J Radiol. 2019;20:894-908. https://doi.org/10.3348/kjr.2018.0540.

13. Levey AS, Bosch JP, Lewis JB, et al. A more accurate method to estimate glomerular filtration rate from serum creatinine: a new prediction equation. Modification of Diet in Renal Disease Study Group. Ann Intern Med. 1999;130:461-70. https://doi.org/10.7326/0003-4819-130-6-19990 3160-00002.

14. Tao J, Zhang W, Wen Y, et al. Endoplasmic reticulum stress predicts clinical response to cyclosporine treatment in primary membranous nephropathy. Am J Nephrol. 2016;43:348-56. https://doi.org/10.1159/000446293.

15. Loupy A, Haas M, Solez K, et al. The banff 2015 kidney meeting report: current challenges in rejection classification and prospects for adopting molecular pathology. Am J Transpl Off J Am Soc Transpl Am Soc Transpl Surg. 2017;17:28-41. https://doi.org/10.1111/ajt.14107.

16. Yamamoto A, Zhang JL, Rusinek $H$, et al. Quantitative evaluation of acute renal transplant dysfunction with low-dose three-dimensional MR renography. Radiology. 2011;260:781-9. https://doi.org/10.1148/radiol.11101 664.

17. Steiger P, Barbieri S, Kruse A, et al. Selection for biopsy of kidney transplant patients by diffusion-weighted MRI. Eur Radiol. 2017;27:4336-44. https://doi.org/10.1007/s00330-017-4814-z.

18. Eisenberger $U$, Binser $T$, Thoeny $H C$, et al. Living renal allograft transplantation: diffusion-weighted MR imaging in longitudinal follow-up of the donated and the remaining kidney. Radiology. 2014;270:800-8. https:// doi.org/10.1148/radiol.13122588.

19. Fan $W$, Ren $T, L i Q$, et al. Assessment of renal allograft function early after transplantation with isotropic resolution diffusion tensor imaging. Eur Radiol. 2016;26:567-75. https://doi.org/10.1007/s00330-015-3841-x.

20. Hueper K, Gutberlet M, Rodt T, et al. Diffusion tensor imaging and tractography for assessment of renal allograft dysfunction-initial results. Eur Radiol. 2011;21:2427-33. https://doi.org/10.1007/s00330-011-2189-0.

21. Lanzman RS, Wittsack H-J, Martirosian P, et al. Quantification of renal allograft perfusion using arterial spin labeling MRI: initial results. Eur Radiol. 2010;20:1485-91. https://doi.org/10.1007/s00330-009-1675-0.

22. Artz NS, Sadowski EA, Wentland AL, et al. Arterial spin labeling MRI for assessment of perfusion in native and transplanted kidneys. Magn Reson Imaging. 2011;29:74-82. https://doi.org/10.1016/j.mri.2010.07.018.

23. Thoeny HC, Zumstein D, Simon-Zoula S, et al. Functional evaluation of transplanted kidneys with diffusion-weighted and BOLD MR imaging: initial experience. Radiology. 2006;241:812-21. https://doi.org/10.1148/ radiol.2413060103.

24. Sadowski EA, Fain SB, Alford SK, et al. Assessment of acute renal transplant rejection with blood oxygen level-dependent MR imaging: initial experience. Radiology. 2005;236:911-9. https://doi.org/10.1148/radiol. 2363041080.

25. Jensen JH, Helpern JA, Ramani A, et al. Diffusional kurtosis imaging: the quantification of non-gaussian water diffusion by means of magnetic resonance imaging. Magn Reson Med. 2005;53:1432-40. https://doi.org/ 10.1002/mrm.20508.

26. Wang W, Yu Y, Wen J, et al. Combination of functional magnetic resonance imaging and histopathologic analysis to evaluate interstitial fibrosis in kidney allografts. Clin J Am Soc Nephrol. 2019;14:1372-80. https:// doi.org/10.2215/CJN.00020119.

27. Rosenkrantz AB, Sigmund EE, Johnson G, et al. Prostate cancer: feasibility and preliminary experience of a diffusional kurtosis model for detection and assessment of aggressiveness of peripheral zone cancer. Radiology. 2012;264:126-35. https://doi.org/10.1148/radiol.12112290.

28. Rosenkrantz AB, Padhani AR, Chenevert TL, et al. Body diffusion kurtosis imaging: basic principles, applications, and considerations for clinical practice. J Magn Reson Imaging. 2015;42:1190-202. https://doi.org/10. 1002/jmri.24985.

29. Sheng RF, Wang $H Q$, Yang $L$, et al. Diffusion kurtosis imaging and diffusion-weighted imaging in assessment of liver fibrosis stage and necroinflammatory activity. Abdom Radiol. 2017;42:1176-82. https://doi. org/10.1007/s00261-016-0984-4.

30. Anderson SW, Barry B, Soto J, et al. Characterizing non-gaussian, high b-value diffusion in liver fibrosis: Stretched exponential and diffusional kurtosis modeling. J Magn Reson Imaging. 2014;39:827-34. https://doi. org/10.1002/jmri.24234.

31. Huang $Y$, Chen $X$, Zhang $Z$, et al. MRI quantification of non-Gaussian water diffusion in normal human kidney: a diffusional kurtosis imaging study. NMR Biomed. 2015;28:154-61. https://doi.org/10.1002/nbm.3235.

32. Pentang $G$, Lanzman RS, Heusch $P$, et al. Diffusion kurtosis imaging of the human kidney: a feasibility study. Magn Reson Imaging. 2014;32:413-20. https://doi.org/10.1016/j.mri.2014.01.006.

33. Sun $K$, Chen X, Chai W, et al. Breast cancer: diffusion kurtosis MR imaging-diagnostic accuracy and correlation with clinical-pathologic factors. Radiology. 2015;277:46-55. https://doi.org/10.1148/radiol.15141625.

34. Liu Y, Zhang G-M-Y, Peng $X$, et al. Diffusional kurtosis imaging in assessing renal function and pathology of IgA nephropathy: a preliminary clinical study. Clin Radiol. 2018;73:818-26. https://doi.org/10.1016/j.crad.2018.05. 012.

35. Zhao J, Wang ZJ, Liu M, et al. Assessment of renal fibrosis in chronic kidney disease using diffusion-weighted MRI. Clin Radiol. 2014;69:1117-22. https://doi.org/10.1016/j.crad.2014.06.011.

36. Xu Y, Zheng $X$, Liu H, et al. Application of diffusional kurtosis imaging for non-invasive assessment of chronic allograft injury. Am J Transpl. 2020. https://doi.org/10.1111/ajt.16171.

\section{Publisher's Note}

Springer Nature remains neutral with regard to jurisdictional claims in published maps and institutional affiliations.

\footnotetext{
Ready to submit your research? Choose BMC and benefit from:

- fast, convenient online submission

- thorough peer review by experienced researchers in your field

- rapid publication on acceptance

- support for research data, including large and complex data types

- gold Open Access which fosters wider collaboration and increased citations

- maximum visibility for your research: over 100M website views per year
}

At BMC, research is always in progress.

Learn more biomedcentral.com/submissions 